\section{Resonating randomness}

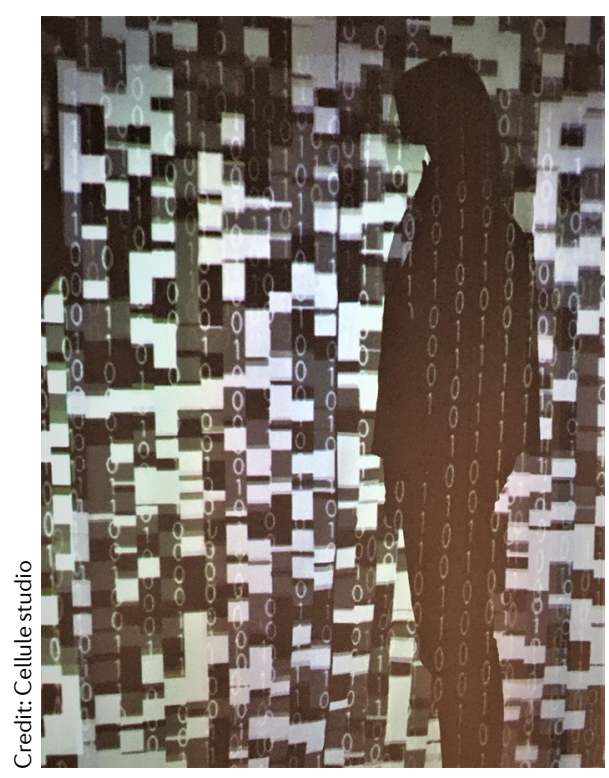

studio Cellule, the installation is an immersive experience, encouraging the audience to engage with the random nature of quantum mechanics.

Turning a corner into the enclosed space of the installation, the first piece is a video. On a black and white kaleidoscopic background with white text running over the top, the viewer is introduced to the idea of quantum randomness governing the internet, our lives and nature. This introduction sets the scene for the science. A resonant tunnelling diode is used to generate a random string of $1 \mathrm{~s}$ and $0 \mathrm{~s}$, which is then projected onto the curved black wall.

At first, the streams of $1 \mathrm{~s}$ and 0 s are mesmerizing, almost comforting. Yet, as I walked in front of the projectors to access the rest of the pieces, squinting in the glare and casting shadows onto the wall, I found myself becoming disorientated. Is this what it feels like inside a computer? At the heart of this installation seems to be the tension between order and chaos. In the popular imagination, science and technology promise efficiency, certainty and order. Quantum mechanics is anything but.

Having been disorientated by the random, but binary, strings of digits on the wall, the viewer is presented with the idea of non-binary quantum superposition. In the centre of the space there are three black boxes; two contain single bulbs which light up (randomly), representing binary states. The box in the middle contains a long cylindrical tube - evoking the dilution fridge which hosts the IBM quantum computer - within which multiple blue bulbs are tangled up, in a physical representation of a probability distribution. This tube seems to light up less frequently, but draws attention when it does, as it is the only use of colour in the exhibition.

\section{$\llbracket$ Is this what it feels like inside a computer?}

On the back wall, an interactive element invites the viewer to join an experiment asking the age-old question "can humans genuinely make random choices?". A black board is littered with white pins, and we are asked to find a spot on the board we like, and add the pin there. Although a few clusters had formed in places, the pins were fairly evenly spread across the board. Perhaps the simplest part of the exhibit, I found this to be the most memorable. Even as a scientist, physically engaging with the act of trying to be random, a feat which quantum systems find effortless, is a powerful reminder of the strangeness of the quantum world.

Reviewed by Ankita Anirban

e-mail:nrmaterials@nature.com

https://doi.org/10.1038/541578-019-0173-5 